Basic Health Sciences

Poster

Abstract ID: 74

\title{
DNA methylation of DISC1 gene in schizophrenia using methylight Taqman assay
}

Nour El Huda Abd Rahim ${ }^{\mathrm{a}}$ | Mohd Nabil Fikri Rahim ${ }^{\mathrm{a}}$ | Norsidah Ku Zaifah ${ }^{\mathrm{a}}$ | Hanisah Mohd Noor ${ }^{\mathrm{b}}$ | Kartini Abdullah ${ }^{\text {b }}$ Norlelawati A. Talib ${ }^{c}$

${ }^{a}$ Department of Basic Medical Sciences, Kulliyyah of Medicine, International Islamic University Malaysia

${ }^{b}$ Department of Psychiatry, Kulliyyah of Medicine, International Islamic University Malaysia

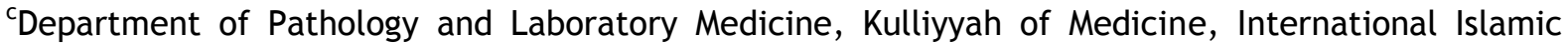
University Malaysia

Introduction: Significant evidences from functional studies have shown that DISC1 gene has a role in the pathogenesis of schizophrenia, although the basis of the genetic defect has yet to be established. There has been a shift of emphasis from DISC1 gene variations to other types of genetic defects namely copy number and epigenetic, both of which have not been well investigated. Thus, the aim of this study is to examine the DNA methylation status of DISC1 gene in patients with schizophrenia. Methods: In this study, 239 subjects were included, 117 schizophrenia patients and 122 healthy controls. Genomic DNA was derived from peripheral blood and bisulfite converted. The DNA methylation level was quantitatively measured by Methylight Taqman analysis. Sociodemographic and the clinical parameters were noted. The severity of the clinical symptoms was assessed using Positive and Negative Syndrome Scale (PANSS). Results: The mean age and gender distribution between the study groups were similar. There was no significant difference in the methylation level of DISC1 between the patients and control group. When patients were compared by age, duration of illness, age at diagnosis, body mass index, smoking status, PANSS score and types of antipsychotic treatment, the DNA methylation level of DISC1, did not show any significant difference $(p>0.05)$. Conclusions: This study found no significant difference in methylation level of DISC1 gene between schizophrenia patients and healthy control. Therefore, it is suggested that aberrant DNA methylation of DISC1 most probably does not contribute to the pathogenesis of schizophrenia.

KEYWORDS: Schizophrenia, DNA methylation, DISC1 